PROCEEDINGS OF THE

AMERICAN MATHEMATICAL SOCIETY

Volume 126, Number 9, September 1998, Pages 2811-2818

S 0002-9939(98)04413-X

\title{
COLLAPSIBLE POLYHEDRA AND MEDIAN SPACES
}

\author{
M. VAN DE VEL \\ (Communicated by Christopher Croke) \\ This paper is dedicated to the memory of my son Wouter, 1974-1993
}

\begin{abstract}
It is shown that a collapsible, compact, connected, simplicial polyhedron admits a cubical subdivision and a median convexity, such that all cubes are convex subspaces with a convexity of subcubes. Conversely, a compact, connected, cubical polyhedron with a convexity as described admits a collapsible simplicial subdivision. Such a convexity, when it exists, is uniquely determined by the corresponding cubical presentation. Some related open problems have been formulated.
\end{abstract}

\section{INTRODUCTION}

Median operators were introduced in the late forties through the work of G. Birkhoff (among others). In a more geometric form, the subject reappeared thirty years later as a study of median convex structures. For a recent illustration of the use of median convexity in polyhedra, the reader may consult Chepoi's paper [4] on facility location. Another interesting application related with optimization (viz., the theory of rectilinear Steiner trees) is described in the author's monograph [15]; it represents joint work with E. Verheul. In this paper, we present a connection between the existence of median convexity in polyhedra and the more classical subject of collapsibility.

Several (partial) characterizations of collapsibility in polyhedra are known. We mention Isbell's criterion for two-dimensional polyhedra (cf. Isbell [6]), and a partial extension to higher dimensional polyhedra (cf. Mai and Tang [8]). The existence of an injective - or hyperconvex - metric (that is, a convex metric such that any collection of closed balls meeting two by two has a common point) is necessary and sufficient in Isbell's result, and is a necessary condition in Mai and Tang's result. Our condition - both necessary and sufficient - is in terms of a related topic: median convexity. The relationship of median(-like) convex structures in metric and normed spaces with questions on injectivity of metrics has been studied by Verheul [16], [17], and is not discussed here. Metric aspects of collapsibility in polyhedra are considered by Verheul [18].

Received by the editors February 22, 1996 and, in revised form, February 5, 1997.

1991 Mathematics Subject Classification. Primary 57Q99, 52A01; Secondary 05C99.

Key words and phrases. Collapsible polyhedron, convex structure, cubical complex, gate map, injective metric, median graph, median operator, simplicial complex.

(C)1998 American Mathematical Society 
In Section 2, we give a brief survey of some notions and results in the area of median convexity. It would lead us too far afield to give full details here; the interested reader is referred to [15]. Section 3 contains the auxiliary results concerning median convexity of polyhedra. The main results are presented in Section 4.

\section{Preliminaries}

By a median algebra is meant a set $X$ with a ternary operator $m: X^{3} \rightarrow X$, such that

(i) (symmetry) $m$ is invariant under permutations of its arguments;

(ii) (idempotence) $m(a, a, b)=b$, and

(iii) (associative or transitive law) for each $b \in X$, the binary operation on $X$, defined by $(u, v) \mapsto u^{*} v=m(b, u, v)$, is associative.

The function $m$ is called a median operator on $X$. A study of median algebras goes back to Birkhoff and Kiss [3] and Sholander [12]; for an extensive survey, see Bandelt and Hedlíková [1]. Isbell [7] describes a somewhat more general notion of median algebra.

There is an equivalent viewpoint of a more geometric flavor: a subset $C$ of a median algebra $X$ is convex provided $m(C \times C \times X) \subseteq C$. The resulting convex structure has two basic properties.

(i) The Kakutani separation property: if $C, D$ are disjoint convex sets, then there is a half-space (that is, a convex set with a convex complement) including $C$ and disjoint with $D$.

(ii) The Helly number is two: if a finite collection of convex sets meet two by two, then these sets meet altogether.

Given three points $a, b, c$ in a convex structure with properties (i) and (ii), one easily verifies that the set

$$
\operatorname{co}\{a, b\} \cap \operatorname{co}\{b, c\} \cap \operatorname{co}\{c, a\}
$$

has exactly one point. Here, "co" denotes convex hull-the smallest convex set including a given set. The resulting ternary function $X^{3} \rightarrow X$ is a median operator. The correspondence between median algebras and convex structures satisfying (i), (ii) is one-to-one. We use the neutral term median space to describe either a median algebra or the equivalent convex structure.

Distributive lattices are a fundamental type of example. The prescription

$$
(a, b, c) \mapsto(a \wedge b) \vee(b \wedge c) \vee(c \wedge a)
$$

yields a median operator and the corresponding median convexity consists of all order-convex sublattices. For a cube $[0,1]^{n}$, this convexity consists exactly of all subcubes (products of closed, half-open, or open segments). It is referred to as the standard median convexity. Each median algebra can be embedded as a medianstable subset of a distributive lattice [15, Cor. I.6.13].

A second class of examples arises in graph theory. If $d$ is a metric on a set $X$ and $a, b, x \in X$, then $x$ is geodesically between $a$ and $b$ provided

$$
d(a, x)+d(x, b)=d(a, b) .
$$

A median graph is a finite connected graph (with an intrinsic geodesic metric $d$, say) such that for each triple of vertices $a, b, c$, there is a unique vertex $x$ which is geodesically in between each pair of the triple. The corresponding ternary function $(a, b, c) \mapsto x$ is indeed a median operator. The simplest example - and a building 
block for all other examples-is the graphic $n$-cube $\{0,1\}^{n}$. This space is also a distributive lattice, but the median operators, derived from the geodesic metric and from the lattice structure, are the same. For a standard reference on median graphs, see Mulder [11].

A recent development is the study of planar regions bounded by a simple closed rectilinear curve. The rectilinear metric of such regions induces a median operator in the same way as the metric of a median graph does. This metric mimics the natural metric of industrial plants. See the paper by Chepoi and Dragan [5].

Throughout, the convex hull of two points $a, b$ is called a segment, and will be denoted briefly by $a b$. The convex hull of a finite set is a polytope. If a convex structure $X$ satisfies the Kakutani separation property and if all singletons are convex, then for each $b \in X$ there is a partial order $\leq_{b}$, defined by

$$
u \leq_{b} v \text { iff } b u \subseteq b v .
$$

In a median convexity there is an abundance of convex sets having a smallest element (a gate) in each base-point order. Such sets are said to be gated. For instance, a nonempty polytope is gated and the gate of an element $c$ in a segment $a b$ is simply $m(a, b, c)$. A gated set $C$ is convex and the resulting gate map

$$
p: X \rightarrow C
$$

is convexity preserving: the inverse image of a convex subset is convex. In fact, the direct $p$-image of a convex set is convex too.

\section{MEdian CONVEXity in CUBICAL POLYHEdRA}

If $X$ is a convex structure and if $Y \subseteq X$, then sets of type $C \cap Y$, where $C$ is a convex set of $X$, are relatively convex in $Y$. The collection of such sets constitutes the relative convexity of $Y$, which turns $Y$ into a subspace of $X$ in the sense of convexity. One of the fundamental geometric results on median spaces is the following (reformulated) "Amalgamation Theorem". Early versions of this result were obtained by Isbell [7] and the author [14].

3.1. Theorem ([15, Thm. 1.5.13, Thm. I.6.14]). Let $X_{1}$ and $X_{2}$ be median spaces such that $X_{1} \cap X_{2}$ is a common, gated, convex subspace of $X_{1}$ and of $X_{2}$. Then there is a unique median convexity on $X_{1} \cup X_{2}$ with the property that $X_{1}$ and $X_{2}$ are convex subspaces. Specifically, a set $C \subseteq X_{1} \cup X_{2}$ is convex iff $C \cap X_{i}$ is convex in $X_{i}$ for $i=1,2$, and $C \cap X_{1} \cap X_{2} \neq \varnothing$ provided $C \cap X_{i} \neq \varnothing$ for $i=1,2$.

By a vertex of a cubical polyhedron is meant a corner point of some cube of the complex. The set of vertices can be turned into a graph - the underlying graph of the polyhedron - by the following prescription. Two vertices form an edge iff they coincide or occur as a pair of end points of an edge in some cube of the complex.

In the next result, the term "subspace" not only covers the usual topological concept; it also refers to convexity. A Hausdorff space with a continuous median operator is a topological median algebra.

3.2. Theorem ([15, Thm. II.3.13, Cor. III.5.25]). Let $P$ be a compact, connected cubical polyhedron with a median convexity such that each cube is a convex subspace with the standard median convexity. Let $G$ be the underlying graph of $P$. Then:

(1) $P$ is a topological median algebra.

(2) $G$ is a median graph and its geodesic convexity is precisely the relative convexity, inherited from $P$. 
(3) The graphic cubes of $G$ are precisely the intersection of cubes in $P$ with $G$.

If $G$ is the underlying graph of a cubical polyhedron and if $C \subseteq G$, then $|C|$ denotes the union of all solid cubes $|Q|$ with $Q \subseteq C$ a graphic cube of $G$.

3.3. Proposition ([15, Lemma II.3.14]). Let $P$ be a compact cubical polyhedron with a median convexity such that each cube is a convex subspace with the standard median convexity. If $C$ is a geodesically convex subset of the underlying graph, then $|C|$ is a convex set of $P$; in fact, $|C|=\operatorname{co}_{P}(C)$.

This leads to the following description of the polyhedral convexity.

3.4. Lemma. Let $P$ be a compact, connected, cubical polyhedron with a median convexity such that each cube is a convex subspace with the standard median convexity. Then a subset of $P$ is convex iff it is connected and it meets each cube in a cubical convex subset.

Proof. By Theorem 3.2(1), $P$ is a topological median algebra. In particular, each segment $a b$ is the image of $P$ under a continuous function

$$
P \rightarrow a b: x \mapsto m(a, b, x) .
$$

It follows that each segment-hence each convex set - of $P$ is connected. By assumption, the intersection of a convex set with a cube is a cubical convex set.

We concentrate on the converse. Let $C \subseteq P$ be a connected set such that $C \cap Q$ is a subcube of $Q$ for each cube $Q$ of $P$. We operate by induction on the minimal number $n$ of cubes covering $P$. For $n=1$, there is nothing left to be proved. Suppose the result to be valid for polyhedra covered with less than $n$ cubes, where $n>1$. By Theorem 3.2, parts (2) and (3), the underlying graph $G$ of $P$ is median and it is not reduced to a single graphic cube. A routine argument shows that $G$ can be obtained as $G_{1} \cup G_{2}$, where $G_{i}$ are (geodesically) convex subsets of $G$ covered with fewer cubes than $G$, and such that each graphic cube of $G$ is in $G_{1}$ or in $G_{2}$. In particular, $\left|G_{1}\right| \cup\left|G_{2}\right|=|G|$ and $\left|G_{1}\right| \cap\left|G_{2}\right|=\left|G_{1} \cap G_{2}\right|$.

By 3.3, the sets $P_{i}=\left|G_{i}\right|$ for $i=1,2$ are convex in $P$ and the set $P_{1} \cap P_{2}$ equals $\left|G_{1} \cap G_{2}\right|$ which, being a polytope, is a gated set. By Theorem 3.1, a subset of $P$ is convex iff it meets both $P_{1}$ and $P_{2}$ in a (possibly empty) convex subset and, if it meets both sets properly, then it meets their intersection. Note that $C$ fulfills the last requirement since $P$ and $C$ are connected. Hence $C$ is convex iff $C \cap P_{i}$ is convex. As $C$ meets each cube of $P_{i}$ in a subcube, the inductive assumption yields that $C \cap P_{i}$ is convex iff it is connected.

Note that the intersection of $C$ with a cube is connected. Therefore, each of the sets $C \cap P_{i}$ can have at most finitely many components. Assume that $\mathcal{C}_{i}$ is the collection of components of $C \cap P_{i}$. If $\mathcal{C}_{1}$ contains two distinct members $D, D^{\prime}$, then, as $\mathcal{C}_{1} \cup \mathcal{C}_{2}$ is a finite closed cover of $C$, there is a sequence of successively intersecting elements of this cover, starting with $D$ and ending with $D^{\prime}$. Note that the members of the chain alternately belong to $\mathcal{C}_{1}$ and $\mathcal{C}_{2}$. Let $D^{\prime \prime}$ be the second element of the chain. Then $D^{\prime \prime}$ is a component of $C \cap P_{2}$ meeting $D$ and some other member of $\mathcal{C}_{1}$. Now $D^{\prime \prime}$ is a connected set which evidently meets each cube of $P_{2}$ in a subcube. It is therefore a convex set. But then $P_{1} \cap P_{2} \cap D^{\prime \prime}$ is a convex-hence connected - set included in $C \cap P_{1}$ and meeting two distinct components of this set, a contradiction. 
3.5. Corollary. Let $P$ be a compact, connected, cubical polyhedron. Then there is at most one median convexity on $P$ such that cubes of $P$ are convex subspaces with the standard median convexity.

It was shown in $[14,5.3]$ that the convexity of a median graph extends uniquely to a median convexity of the realizing cubical polyhedron, such that cubes are convex subspaces with the standard median convexity and the realization of a graphical convex set is a polyhedral convex set. It appears from the previous results that the second condition is, in fact, redundant.

3.6. Corollary. Let $P_{1}, P_{2}$ be polyhedra with a median convexity such that each cube is a convex subspace with the standard median convexity, and let $f: G_{1} \rightarrow G_{2}$ be a convexity preserving function between the respective underlying median graphs $G_{i}$ of $P_{i}$. Then the canonical piece-wise linear extension $|f|: P_{1} \rightarrow P_{2}$ of $f$ is convexity preserving.

Proof. Let $C \subseteq P_{2}$ be convex. By Lemma 3.4, it suffices to show that for each graphic cube $Q$ of $P_{1}$ the set

$$
|f|^{-1}(C) \cap|Q|
$$

is convex. To verify the latter, note that $f(Q)$ is a graphic cube of $P_{2}$. Hence $|f|(|Q|)=|f(Q)|$. As $C \cap|f(Q)|$ is a subcube of a cube, the result follows.

\section{The MAIN Results}

4.1. Simplicial and cubical collapsing. Let $S$ be a simplicial complex. An elementary collapse of $S$ is a subcomplex $S^{\prime}$ of $S$ such that there is a simplex $\sigma$ of $S$, together with a facet $\tau$ of $\sigma$, such that neither $\sigma$ nor $\tau$ are in $S^{\prime}$ and $S$ obtains from $S^{\prime}$ by adding $\sigma$ and $\tau$ to it. Observe that $\tau$ is a facet of only one simplex of $S$ (a free face). The simplicial complex $S$ (or, the polyhedron represented by it) is collapsible if there is a sequence of elementary collapses transforming $S$ into a one-point complex. Stallings [13] is a standard reference.

Let $P$ be a cubical polyhedron. A cuboid in $P$ is a cubical subcomplex $L$ which is connected and meets each cube in a subcube (which must then be a face of the given cube). By an elementary collapse of $P$ is meant a cubical subcomplex $P^{\prime}$ of $P$ such that there is a cuboid $L \subseteq P^{\prime}$ and an isomorphism of cubical complexes

$$
P^{\prime} \cup(L \times[0,1]) \approx P .
$$

It is understood that $L \times[0,1]$ is built exclusively with cubes of type $Q \times\{0\}$, $Q \times\{1\}$, and $Q \times[0,1]$, where $Q$ is a cube of $L$. Following Mai and Tang [8], $P$ is (cubically) collapsible provided there is a sequence of elementary collapses taking $P$ into a one-point complex. By Lemma 1 of Mai and Tang, a compact, simplicially collapsible polyhedron admits a cubical refinement which is cubically collapsible. The idea behind this result is the following. Let $S, S^{\prime}, \sigma, \tau$ be as in the definition of elementary collapse. Then there is a homeomorphism $h$ between $(\partial \sigma \backslash \operatorname{Int}(\tau)) \times[0,1]$ and $\sigma$ such that $h(x, 0)=x$ for each $x \in \partial \sigma \backslash \operatorname{Int}(\tau)$. Given a collapsible cubical refinement $M^{\prime}$ of $S^{\prime}$, let $L$ be the subcomplex of $M^{\prime}$ on the set $\partial \sigma \backslash \operatorname{Int}(\tau)$. Then use $h$ to produce a cubical refinement $M^{\prime} \cup L \times[0,1]$ of $S$. It is not difficult to verify that a collapsible cubical complex has a collapsible simplicial refinement. The basic step of the argument goes as follows. Let $L$ be a cubical complex and let $S$ be any simplicial subdivision of $L$. Then the (usual) simplicial presentation of $S \times[0,1]$ is a refinement of $L \times[0,1]$ and $S \times[0,1]$ collapses simplicially to $S \times\{0\}$. 
We will now show that-roughly stated-collapsibility is equivalent with the existence of a median convex structure as described in the previous section.

4.2. Theorem. A compact, collapsible simplicial polyhedron $P$ has a cubical presentation refining the given simplicial presentation, together with a median convexity on $P$, such that each cube of the cubical presentation of $P$ is a convex subspace with the standard median convexity. Conversely, if a cubical polyhedron $P$ has a median convexity as described, then $P$ is cubically collapsible; in fact, there is a collapsible simplicial presentation refining the given cubical presentation.

Proof. Assume first that $P$ is a collapsible simplicial polyhedron. By a result of Mai and Tang quoted above, there is a cubical refinement of the simplicial structure which is cubically collapsible. We establish the result by induction on the number $n$ of cubes involved in this refinement. For $n=1, P$ is a one-point complex. Assume that $n>1$ and that the result holds for polyhedra with fewer cubes. Consider a sequence of elementary collapses of $P$ into a one-point complex. The first step $P^{\prime}$ in this sequence is itself a collapsible polyhedron with fewer cubes. By inductive assumption, there is a median convexity on $P^{\prime}$ such that each cube is a convex subspace with the standard median convexity. Let $L \subseteq P^{\prime}$ be the cuboid involved in the first elementary collapse. By Lemma 3.4, $L$ is a convex set of $P^{\prime}$. We consider the relative convexity of $L$ and the natural convexity of $[0,1]$. Both are median convexities. Then $L \times[0,1]$ can be given the product convexity, which is evidently median. Observe that $L$ occurs as a polytope in the spaces $P^{\prime}$ and $L \times[0,1]$. In particular, $L$ is gated. We are in a position to apply Theorem 3.1: There is a median convexity on $P \approx P^{\prime} \cup(L \times[0,1])$ such that $P^{\prime}$ and $L \times[0,1]$ are convex subspaces. Since each cube of $P$ is a cube of $P^{\prime}$ or of $L \times[0,1]$, it follows that the resulting convexity of $P$ is as required.

We next establish the converse. Let $P$ be a compact connected cubical polyhedron with a median convexity such that each cube of $P$ is a convex subspace with the standard median convexity. Let $G$ be the underlying median graph of $P$; cf. Theorem 3.2. We assume that $G$ is not a one-point graph. Let $H \subset G$ be a minimal proper half-space and let $H^{\prime}$ be its complement. As $G$ is finite, there are gate functions

$$
p^{\prime}: G \rightarrow H^{\prime} ; \quad p: G \rightarrow H .
$$

If $p\left(H^{\prime}\right)$ is not the whole of $H$, say: $x \in H \backslash p\left(H^{\prime}\right)$, then consider a half-space $H_{0}$ of $G$ with $x \in H_{0}$ and $p\left(H^{\prime}\right) \cap H_{0}=\varnothing$. By the elementary properties of gates, $p$ maps $H^{\prime} \cap H_{0}$ into $H_{0}$, which conflicts with our assumptions unless $H^{\prime} \cap H_{0}=\varnothing$. But then $H_{0}$ is included properly in $H$, a contradiction.

We next use the fact [15, Prop. I.5.11(4)] that $p \circ p^{\prime} \circ p$ equals $p$ on $H^{\prime}$. As $p\left(H^{\prime}\right)=H$, it follows that $p \circ p^{\prime}$ is the identity on $H$. Hence $p^{\prime}$ maps $H$ bijectively onto a convex subset $p^{\prime}(H)$ of $H^{\prime}$. Consequently, $p^{\prime}$ is an isomorphism between the median spaces $H, p^{\prime}(H)$. On the other hand [14], the subspace $H \cup p^{\prime}(H)$ of $G$ is convex and its relative convexity corresponds with the product convexity of $p^{\prime}(H) \times\{0,1\}$ (where the second factor is given the "discrete" convexity). It follows easily that there is an isomorphism

$$
G \approx H^{\prime} \cup\left(p^{\prime}(H) \times\{0,1\}\right) .
$$

Its piece-wise linear extension is an isomorphism of cubical complexes

$$
P \approx\left|H^{\prime}\right| \cup\left(\left|p^{\prime}(H)\right| \times[0,1]\right)
$$


(cf. 3.6). Note that $p^{\prime}(H)$ is a cuboid by Lemma 3.4. This shows that $P$ collapses to $\left|H^{\prime}\right|$. As $\left|H^{\prime}\right|$ is a cubical complex with a median convexity satisfying the usual condition on cubes, we can proceed by induction to obtain a continued sequence of elementary cubical collapses ending at a one-point complex.

4.3. Problems and comments. Let $X$ be a topological median algebra on a cubical polyhedron. Is it possible to construct a new median convexity on $X$ which is compatible with some refinement of the cubical presentation of $P$ ? Here, "compatibility" is understood as in Theorem 4.2. It seems to be unknown whether collapsibility is a topological property of polyhedra, although certain closely related conditions are not (e.g., M. E. Rudin's example of a non-shellable triangulation of a 3-cell; cf. Bing [2]).

Is it true that a compact connected polyhedron $P$ is collapsible if and only if it admits a continuous median operator such that $P$ is the convex hull of its vertex set and every two of its vertices are connected by a piece-wise convex and piece-wise linear path? More generally, is it true that a compact connected polyhedron $P$ is collapsible if and only if it admits a continuous median operator? (Note that this is a topological property.)

Some of the conditions occurring in these questions require further explanation. By a dendron is meant a connected compact Hausdorff space in which each pair of distinct points can be separated by the removal of a third point. Van Mill and Schrijver [9] showed that the connected subsets of a dendron constitute a median convexity. Van Mill and van de Vel [10] showed that the resulting continuous median operator is unique. A metrizable dendron with infinitely many branches provides an example of a compact, metrizable and connected median space of finite dimension which is not polyhedral.

In [15], a method has been outlined to construct a median convexity on $[0,1]^{2}$, in which a convex subspace occurs which is a prescribed dendron with finitely many branches. It is easy to extend this method with the aid of inverse limits to produce convex dendra with countably many branches. Although the underlying space is a polyhedron, there can be no cubical presentation such that each cube is a convex subspace with the standard median convexity.

The standard median convexity of a cube $Q$ can be characterized up to isomorphism as follows [15, II.3.23(1)].

(1) $Q$ is the hull of its corner points.

(2) Each edge of $Q$ is a convex set and its relative convexity is the natural one.

In particular, every two corner points can be joined by a piece-wise convex and piece-wise linear path.

Finally, the following question has been suggested by the referee. Is it possible to define a cubical refinement of a given simplicial complex $S$ such that $S$ is collapsible if and only if the obtained cubical complex is median? The cubical complex proposed by Mai and Tang heavily depends on the sequence of elementary collapses of $S$.

\section{REFERENCES}

1. H.-J. Bandelt, J. Hedlíková, Median algebras, Discrete Math., 45 (1983), 1-30. MR 84h:06015

2. R. H. Bing, The geometric topology of 3-manifolds, Amer. Math. Soc. Coll. Publ. 40, Amer. Math. Soc., Providence, R.I., (1983), x+238 pp. MR 85j:57001 
3. G. Birkhoff, S. A. Kiss, A ternary operator in distributive lattices, Bull. Amer. Math. Soc., 53 (1947), 749-752.

4. V. D. Chepoi, A multifacility location problem on median spaces, Discrete Math. Appl., 64 (1) (1996), 1-29. MR 97c:90058

5. V. D. Chepoi, F. F. Dragan, Computing a median point of a simple rectilinear polygon, Inform. Process. Lett., 49 (1994), 281-285.

6. J. R. Isbell, Six Theorems about injective metric spaces, Comment. Math. Helv., 39 (1964), 65-76. MR 32:431

7. J. R. Isbell, Median algebra, Trans. Amer. Math. Soc., 260 (1980), 319-362. MR 81i:06006

8. J.-H. Mai, Y. Tang, An injective metrization for collapsible polyhedra, Proc. Amer. Math. Soc., 88 (1983), 333-337. MR 84g:54036

9. J. van Mill, A. Schrijver, Subbase characterization of compact topological spaces, Gen. Top. Appl., 10 (1979), 183-201. MR 80d:54023

10. J. van Mill, M. van de Vel, Convexity preserving mappings in subbase convexity theory, Proc. Kon. Ned. Akad. Wet., A 81 (1978), 76-90. MR 80f:52014

11. H. M. Mulder, The interval function of a graph, Math. Centre Tracts 132, Mathematisch Centrum, Amsterdam (1980). MR 82h:05045

12. M. Sholander, Trees, lattices, order, and betweenness, Proc. Amer. Math. Soc., 3 (1952), 369-381. MR 14:9b

13. J. R. Stallings, Lectures on polyhedral topology, Tata Institute of Fundamental Research, Bombay (1967). MR 38:6605

14. M. van de Vel, Matching binary convexities, Top. Appl., 16 (1983), 207-235. MR 85f:52026

15. M. van de Vel, Theory of convex structures, Elsevier Science Publishers, Amsterdam (1993), 540+xv pp. MR 95a:52002

16. E. R. Verheul, Modular metric spaces, Report WS-358 (1989).

17. E. R. Verheul, Modular normed spaces, Report WS-365 (1990).

18. E. R. Verheul, Multimedians in metric and normed spaces, CWI tract 91, Centrum voor Wiskunde en Informatika, Amsterdam, Netherlands (1993). MR 94i:54062

Fakulteit Wiskunde en Informatika, VRije Universiteit, NL-1081 HV Amsterdam, the NETHERLANDS

E-mail address: marcel@cs.vu.nl 